\title{
miR-203 Suppresses Tumor Growth and Angiogenesis by Targeting VEGFA in Cervical Cancer
}

\author{
Xiangyu Zhu ${ }^{\mathrm{a}, \mathrm{c}}$ Kejun Erb,c Caiying Mao ${ }^{\mathrm{a}}$ Qi Yan ${ }^{\mathrm{a}}$ Haijun $\mathrm{Xu}^{\mathrm{a}}$ Yuanbei Zhang ${ }^{\mathrm{a}}$ \\ Jianhong Zhu ${ }^{\mathrm{a}}$ Fang Cui ${ }^{\mathrm{a}}$ Wenxia Zhao ${ }^{\mathrm{a}}$ Hong Shi ${ }^{\mathrm{a}}$ \\ a'Department of Gynecology and Obstetric Medicine, Shanghai Jiangwan Hospital, Shanghai, P. R. China; \\ 'Department of Gynecology, Shanghai First People's Hospital Branch, Shanghai, PR China; 'These authors \\ contributed equally to the study
}

\section{Key Words}

miR-203 • Cervical cancer • VEGFA

\begin{abstract}
Background/Aims: MicroRNA (miRNA) plays important roles in the development of different cancers. In this study, we investigated the roles and mechanisms of miR-203 in human cervical cancer. Methods: miR-203 expression was detected in cervical cancer tumors and cell lines by qRT-PCR. The methylation status in the promoter region of miR-203 was examined by methylation-specific PCR. The functional effect of miR-203 was determined by both in vitro and in vivo assays. Results: miR-203 was frequently down-regulated in cervical cancer tumors and cell lines. This down-regulation of miR-203 was associated with methylation of the miR-203 promoter. Furthermore, miR-203 down-regulated vascular endothelial growth factor alpha (VEGFA) expression by directly targeting its 3 '-untranslated region. Functional assays revealed that miR-203 suppressed cervical cancer cell proliferation, tumor growth, and angiogenesis in nude mice, whereas forced expression of VEGFA rescued this inhibitory effect. Conclusion: Our collective findings indicate that miR-203 functions as a tumor suppressor by targeting VEGFA, resulting in the inhibition of tumor growth and angiogenesis. Thus, miR-203 may be a potential therapeutic target and prognostic marker in cervical cancer.
\end{abstract}


Zhu et al.: miR-203 in Cervical Cancer

\section{Introduction}

Cervical cancer is highly prevalent and the leading cause of cancer death in women worldwide [1]. Concomitantly, the incidence and mortality of cervical cancer are rising, but currently there is no effective chemoprevention or treatment. Therefore, new and effective treatment approaches are necessary to reduce mortality and extend survival of cervical cancer patients.

MicroRNAs (miRNAs) are a recently discovered class of small noncoding RNA molecules that modulate gene expression [2]. Accumulating evidence has indicated that miRNAs are master regulators of several important biological processes, including cell apoptosis, proliferation, migration, invasion, and metabolism [3-6]. Several miRNAs are down-regulated in cervical cancer, such as miR-214, miR-143, miR-375 and miR-23b [7$10]$, suggesting their potential involvement in the development of this malignancy. Recently, miR-203 has been reported to be deregulated in cervical cancer compared with normal cervical squamous epithelial specimens, and its downregulation is closely associated with promoter hypermethylation [11-13]. The involvement of miR-203 in the tumorigenesis and development of bladder, breast, and prostate cancers, as well as hepatocellular carcinoma have also been reported [14-17]. However, the underlying mechanisms by which miR-203 affects tumor growth and angiogenesis in cervical cancer remain largely unknown.

In the present study, we found that miR-203 functions as a tumor suppressor by directly targeting vascular endothelial growth factor alpha (VEGFA). We confirmed that miR-203 is down-regulated in cervical cancer because of promoter hypermethylation. Also, miR-203 overexpression inhibited VEGFA both at the mRNA and protein levels, suppressed cell proliferation, and attenuated tumor growth and angiogenesis in nude mice. Thus, our findings provide significant clues regarding the role of miR-203 as a tumor suppressor in cervical cancer.

\section{Materials and Methods}

\section{Cell culture and tumor specimens}

Five human cervical cancer cell lines (HeLa, C33A, SiHa, CaSki and HT-3) were purchased from the American Typed Cell Culture (Manassas, VA, USA) and grown in Dulbecco's modified Eagle's medium with $10 \%$ fetal bovine serum at $37{ }^{\circ} \mathrm{C}$ in an atmosphere of $5 \% \mathrm{CO}_{2}$. A total of 34 pairs of human cervical cancer tissues and adjacent normal cervical tissues were collected from Shanghai Jiangwan Hospital (Shanghai, China). Specimens were obtained with informed patient consent and the study was approved by the Ethical Committee of Shanghai Jiangwan Hospital.

RNA extraction and quantitative real-time PCR ( $q R T-P C R$ ) analysis

Total RNA for mRNA and miRNA analyses was extracted using TRIzol reagent (Invitrogen, Carlsbad, CA, USA) following the manufacturer's instructions. Complementary DNA (cDNA) was synthesized from $2 \mu \mathrm{g}$ of total RNA using the PrimeScript RT Reagent Kit (TaKaRa, Otsu, Shiga, Japan). To quantify mature miR-203 expression, stem-loop qRT-PCR was performed using an ABI PRISM 7500 Sequence Detection System (Applied Biosystems, Inc., Foster City, CA, USA) with the TaqMan ${ }^{\circledR}$ MicroRNA Reverse Transcription Kit (Applied Biosystems). RNU6B was used as an endogenous control for normalization. qRT-PCR analyses for VEGFA and the normalization control gene glyceraldehyde 3-phosphate dehydrogenase (GAPDH) were performed using SYBR Premix Ex Taq (TaKaRa) on an ABI PRISM 7500 Sequence Detection System (Applied Biosystems). The relative expression of each gene was calculated and normalized using the $2^{-\Delta \Delta \mathrm{Ct}}$ method relative to RNU6B or GAPDH.

\section{5-aza-2'-deoxycytidine treatment}

$\mathrm{SiHa}$ and CaSki cells were seeded at $2 \times 10^{5}$ cells per $10-\mathrm{cm}$ dish $24 \mathrm{~h}$ prior to treatment with $5 \mu \mathrm{M}$ 5-aza-2'-deoxycytidine (Aza, Sigma-Aldrich Corporation, St. Louis, MO, USA). Cells were incubated for $72 \mathrm{~h}$ with a change of culture medium every $24 \mathrm{~h}$. 
Zhu et al.: miR-203 in Cervical Cancer

DNA methylation analysis

DNA methylation was determined using PCR analysis by converting $1 \mu \mathrm{g}$ of genomic DNA (gDNA) with sodium bisulfite, as previously reported [18]. Then, methylation-specific PCR (MSP) was performed using primers specific for either the methylated or modified unmethylated DNA. The methylation status was also analyzed by bisulfite genomic sequencing (BGS) of both strands of the corresponding CpG islands, as described previously [19]. The MSP primer sequences for the miR-203 promoter were 5'-GGGTTGTGGAGGATTAGTT-3' (forward) and 5'-AAACAACTAAACTCCAAACA-3' (reverse) for unmethylated reactions, and 5'-GGGTCGTGGAGGATTAGTC-3' (forward) and 5'-AAACGACTAAACTCCGAACG-3' (reverse) for methylated reactions. The BGS primers were 5'-GAATTCGGGAGGTTAGGTG-3' (forward) and 5'-ACCCCCTACCCTACTACAACC-3' (reverse).

\section{Lentiviral infection and oligonucleotide transfection}

The human miR-203 precursor or anti-miR203 (Ambion, Austin, TX, USA) was cloned into the lentivirus-based expression plasmid pLenti-6.3 (Invitrogen). The primers for pre-miR-203 were 5'-GAATTCGGG GATCTGGGCGCAGGGGCC-3' (forward) and 5'-CTCGAGCCGACCTGGAGCGCGGAGC-3' (reverse). Viral packaging and infection were performed according to standard protocols as recommended by the manufacturer. Cells $\left(1 \times 10^{6}\right)$ were infected with $1 \times 10^{7}$ lentivirus transducing units in the presence of $10 \mu \mathrm{g} / \mathrm{ml}$ polybrene (Sigma-Aldrich). An empty lentiviral vector was used as negative control.

\section{Plasmid construction and luciferase reporter assays}

The full-length open reading frame of VEGFA was cloned into pcDNA3.1 (+) to generate VEGFA expression vectors. The primers for VEGFA were 5'-ACCATGAACTTTCTGCTGTCTTGGGTGCAT-3' (forward) and 5'-TCACCGCCTCGGCTTGTCACATCTGCAAGT-3' (reverse). The wild-type 3'UTR (WT) of VEGFA (from 1101 to $1419 \mathrm{bp}$ in the 3'UTR of VEGFA) was cloned into Xho I/Hind III sites of the pGL3 Basic Vector (Promega, Madison, WI, USA). Site-directed mutagenesis of the miR-203 seed sequence in the VEGFA 3'UTR (Mut) was performed using the QuikChange ${ }^{\mathrm{TM}}$ Site-Directed Mutagenesis Kit (Stratagene, La Jolla, CA, USA). The primers for VEGFA 3'-UTR were 5'-CAGCTCGAGTGTGTGAGTGGTTGACCTTCCT-3' (forward) and 5'-CCGAAGCTTTCAGGGAGAGAGAGATTGGAAA-3' (reverse). For the reporter assays, stable miR-203 overexpressing clones were transiently transfected with WT or Mut reporter plasmids using Lipofectamine 2000 reagent (Invitrogen). After $48 \mathrm{~h}$, the cells were harvested and lysed, and luciferase activity was measured using the Dual-Luciferase Reporter Assay System (Promega). Renilla-luciferase was used for normalization.

\section{Cell proliferation assay}

For the cell proliferation assays, SiHa and CaSki cells $\left(7 \times 10^{3}\right.$ cells/well $)$ overexpressing miR-203 or miR-control were separately seeded in 96-well plates. At $24,48,72,96$, and $120 \mathrm{~h}$, cell viability was assessed using the MTT (3-(4,5-dimethylthiazol-2-yl)-2,5-diphenyltetrazolium bromide) assay as previously described [20].

\section{Western blot analysis}

The cells were lysed with radioimmunoprecipitation assay lysis buffer and total protein content was collected by centrifugation. Lysate proteins $(20 \mu \mathrm{g})$ were separated by sodium dodecyl sulfate polyacrylamide gel electrophoresis and subsequently transferred to a nitrocellulose membrane, which was blocked with $5 \%$ non-fat dry milk for $2 \mathrm{~h}$ and incubated with primary antibodies against VEGFA (Cell Signaling Technology, Inc., Danvers, MA, USA) and GAPDH (Santa Cruz Biotechnology, Inc., Santa Cruz, CA, USA). Protein bands were detected by incubation with horseradish peroxidase-conjugated antibodies and visualized with an enhanced chemiluminescence reagent (Amersham Biosciences, Piscataway, NJ, USA).

\section{Tumorigenicity assay in nude mice}

Animal experiments were performed according to our institutional guidelines on the use and care of animals. Athymic BALB/c nude mice (4 weeks old) were randomly assigned to four groups (8 mice/ group). SiHa-miR-203 or HeLa-anti-miR203 and the corresponding control cells were trypsinized and resuspended at a final concentration of $5 \times 10^{6}$ cells $/ 0.1 \mathrm{ml}$ in phosphate-buffered saline. Cells were injected subcutaneously into the right flanks of the nude mice, which were observed over 4 weeks for tumor 

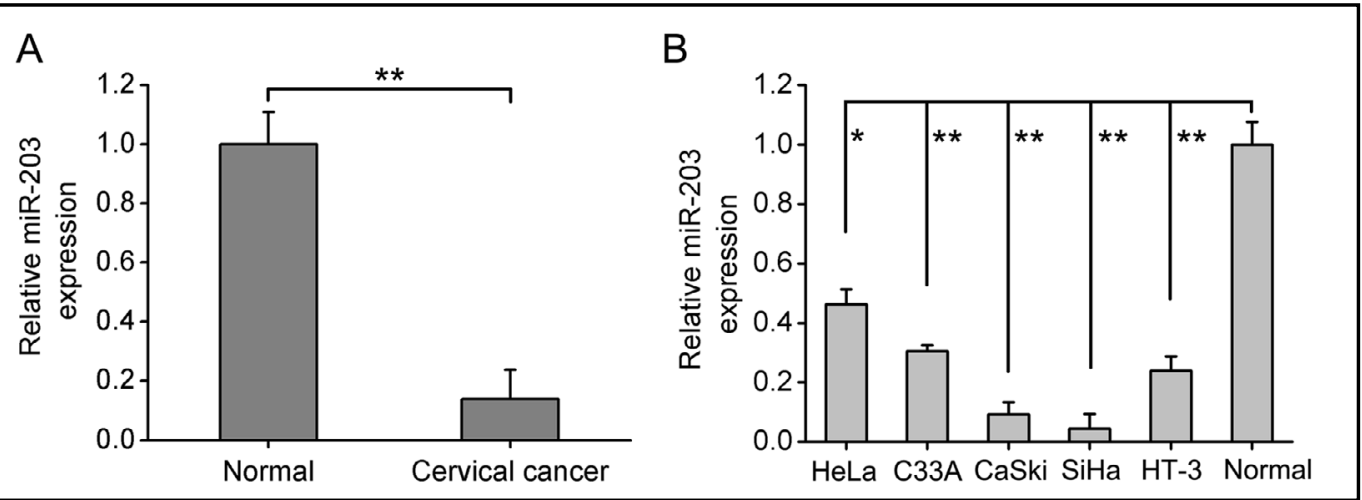

Fig. 1. miR-203 expression in human cervical cancer tissues and cell lines. (A) miR-203 expression levels in 34 pairs of cervical cancer tissues and adjacent normal tissues were measured by qRT-PCR with RNU6B levels as an internal control. (B) The expression levels of miR-203 were analyzed by qRT-PCR in cervical cancer cell lines (HeLa, C33A, CaSki, SiHa, and HT-3). Results represent the mean ratio between miR-203 and RNU6B from 3 experiments. ${ }^{*} p<0.05$ and ${ }^{* *} p<0.01$.

formation. Tumor growth was monitored by measuring the largest (a) and smallest (b) perpendicular diameters with calipers, and calculating the tumor volume $(\mathrm{V})=\mathrm{a} \times \mathrm{b}^{2} \times 0.5$. After 25 days, the mice were sacrificed and tumors were recovered, fixed and stained for angiogenesis.

\section{Immunohistochemical analysis}

Tumors were fixed with $4 \%$ paraformaldehyde for $24 \mathrm{~h}$, washed with $70 \%$ ethanol, and processed by the paraffin-embedded method. The tissues sections ( $5 \mathrm{~mm}$ thick) were then heat-immobilized or pepsinimmobilized according to the manufacturer's instructions. Antibodies against CD31 (Abcam, Cambridge, MA) were used for the immunostaining, followed by incubation with anti-rabbit secondary antibodies, and visualized with Envision System (DAKO Corporation, Carpinteria, CA, US) and diaminobenzadine (DAB kit, Invitrogen). Microvessel density was evaluated by counting 5 random high power fields (200x).

\section{Statistical analysis}

Statistical analysis was performed using SPSS v13.0 statistical software (SPSS, Inc., Chicago, IL, USA) and data were expressed as the mean \pm standard error of at least three independent experiments. Student's $t$-test was used to analyze differences between two groups and Spearman's correlation analysis was used to analyze the correlation between miR-203 and VEGFA mRNA expression. Differences were considered statistically significant at a $p$-value $<0.05\left({ }^{*} p<0.05\right.$ and $\left.{ }^{* *} p<0.01\right)$.

\section{Results}

miR-203 is down-regulated in cervical cancer

Previous studies showed that miR-203 expression was significantly reduced in cervical cancer. To confirm these results, qRT-PCR analysis was performed in 34 paired samples of cervical cancer. As shown in Fig. 1A, miR-203 was strongly down-regulated in cervical cancer tissues compared to adjacent normal cervical tissues. Similarly, miR-203 expression was generally lower in cervical cancer cell lines than normal cervical tissues (Fig. 1B). Taken together, these results suggest that miR-203 is down-regulated in human cervical cancer and cell lines.

miR-30a down-regulation is caused by promoter hypermethylation

To confirm that miR-203 down-regulation is caused by promoter hypermethylation in cervical cancer, the methylation status of the miR-203 promoter was tested by MSP. Full or partial methylation was observed in five cervical cancer cell lines (HeLa, C33A, CaSki, SiHa, and HT-3). This was accompanied by down-regulated miR-203 expression, whereas miR-203 


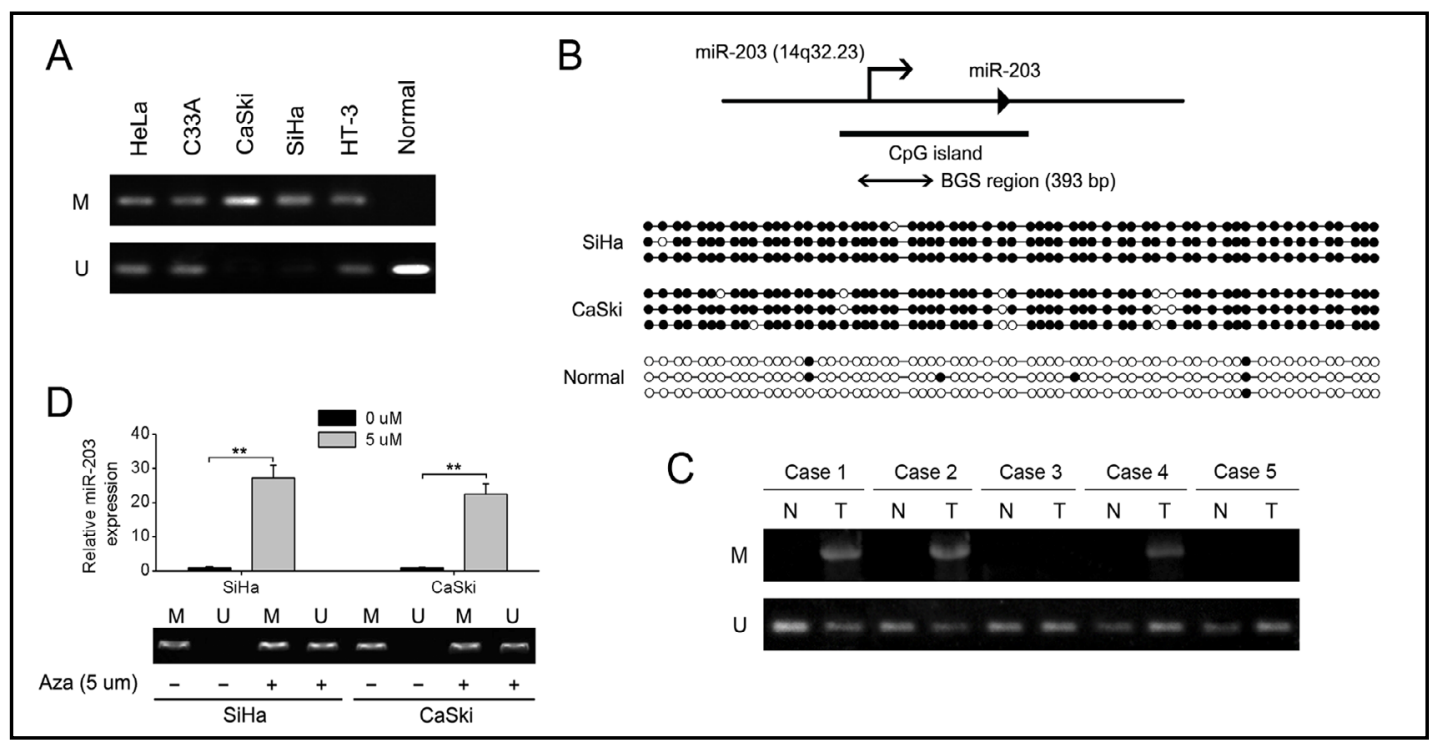

Fig. 2. Hypermethylation of the miR-203 promoter region in cervical cancer cell lines. (A) MSP analysis of the miR-203 promoter region in five cervical cancer cell lines. (B) Schematic representation of the miR-203 gene embedded in a CpG island analyzed for methylation status. BGS analysis of the miR-203 promoter region in cervical cancer cell lines $\mathrm{SiHa}$, CaSki, and normal cervical tissue (three clones for each sample). Black and white circles represent methylated and unmethylated CpG, respectively. (C) MSP analysis was performed on normal cervical tissue and cervical cancer tissues. (D) Demethylation treatment with $5 \mu \mathrm{M}$ 5-aza-2'-deoxycytidine (Aza) restored miR-203 expression in SiHa and CaSki cells. The relative expression of miR-203 in SiHa and CaSki cells treated with Aza was shown in the upper panel. Results represent mean \pm standard error of the miR-203/RNU6B ratio normalized to the baseline level in the SiHa or CaSki scramble control cells $(\mathrm{n}=3) .{ }^{*} p<0.05$ and ${ }^{* *} p<0.01$. MSP analysis of the miR-203 promoter region was shown in the lower panel. M, methylated; U, unmethylated.

promoter methylation was not detected in the normal cervical tissues (Fig. 2A). In addition, BGS was used to characterize miR-203 promoter methylation in the SiHa and CaSki cell lines and normal cervical tissues. Dense methylation was found in SiHa and CaSki cells, but not in normal cervical tissues (Fig. 2B). Consistent with these observations, MSP analysis showed that the miR-203 promoter was hypermethylated in 44.7\% (9 of 34) of tumour specimens, unmethylated in all normal cervical tissues (Fig. 2C). To further confirm these results, SiHa and CaSki cells were treated with the demethylation agent 5-aza-2'-deoxycytidine (Aza). This restored miR-203 expression (Fig. 2D), indicating that miR-203 down-regulation was mediated by promoter methylation in cervical cancer cells.

The VEGFA 3'-UTR is a target of miR-203

To explore the function of miR-203, three computational algorithms (TargetScan, PicTar, and miRanda) were used to search for potential miR-203 target genes, especially for those involved in tumor growth and angiogenesis. Basing on this rationale, 5 candidate genes (VEGFA, FGF2, PDGFRA, E2F3 and AKT2) were selected. We were particularly interested in VEGFA because of its positive roles in tumor growth and angiogenesis [21, 22]. Fig. 3A showed that the VEGFA 3'-UTR contained a complementary site for the miR-203 seed region, which was conserved across species. To confirm that VEGFA is a direct target of miR-203, we subcloned a 3'-UTR fragment of VEGFA (WT) containing the binding site into the pGL3 reporter vector. As shown in Fig. 3B, miR-203 overexpression in SiHa and CaSki cells, markedly suppressed the luciferase activity of the WT reporter, while this profound inhibition was abolished when the target miR-203 seed sequences were mutated (Mut) or by tansfection with anti-miR203. Accordingly, miR-203 inhibition by the addition of antimiR203 in HeLa cells rescued luciferase expression (Fig. 2C). To directly assess the effect of 


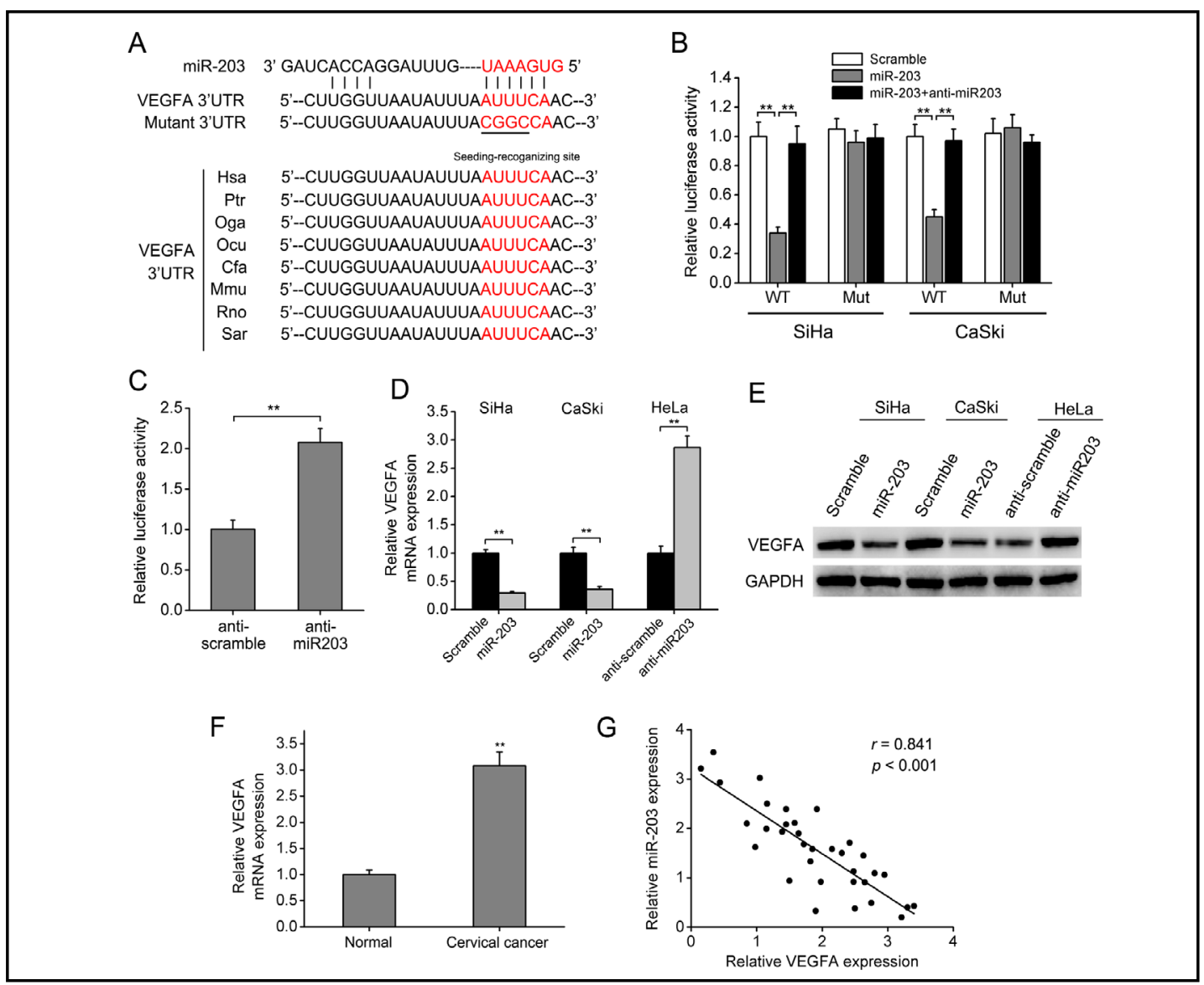

Fig. 3. VEGFA is a downstream gene target of miR-203. (A) Sequence alignment of miR-203 and the VEGFA 3'-UTR, which contained one predicted miR-203-binding site. The miR-203 seed regions and the seed-recognizing sites in the VEGFA 3'-UTR are indicated in red. All nucleotides in the seed-recognizing sites were completely conserved in several species. (B) Luciferase assay analysis of SiHa and CaSki cells co-transfected with miR-203 or/and anti-miR203, and a luciferase reporter containing VEGFA 3'-UTR (WT) or a mutant (Mut). Luciferase activities were measured $48 \mathrm{~h}$ post-transfection. Data are presented as mean \pm standard error from 3 independent experiments. (C) Luciferase assay analysis of HeLa cells co-transfected with a luciferase reporter and anti-miR203. Inhibition of endogenous miR-203 rescued the luciferase expression. Data are presented as mean \pm standard error from 3 independent experiments. (D) VEGFA mRNA levels were analyzed following miR-203 or anti-miR203 transfection by qRT-PCR. Data are presented as mean \pm standard error from 3 independent experiments. (E) miR-203 anti-miR203 transfection affects VEGFA protein levels. (F) Relative expression levels of VEGFA in 34 cervical cancer and adjacent normal cervical samples were assayed. (G) Correlation of VEGFA expression to miR-203 expression in 34 cervical cancer samples using Spearman's correlation analysis. ${ }^{*} p<0.05$ and ${ }^{* *} p<0.01$.

miR-203 on VEGFA expression, miR-203 was stably overexpressed in SiHa and CaSki cells using lentiviral expression vectors, as these cells have low basal levels of miR-203 among cervical cancer cell lines (Fig. 1B). As shown in Figs. 3D and E, miR-203 overexpression down-regulated VEGFA expression at both the mRNA and protein levels. In contrast, miR203 knockdown using anti-miR203 in HeLa cells, which have high endogenous miR-203 levels, significantly increased VEGFA expression. These results provide evidence that miR203 directly recognizes the 3'-UTR of VEGFA mRNA and inhibits its expression.

VEGFA levels are up-regulated in cervical cancer tissues and inversely correlated with miR-203 expression

To determine whether reduced miR-203 expression is correlated to VEGFA expression levels in tumor tissues, we evaluated VEGFA expression in 34 cervical cancer and adjacent 
A

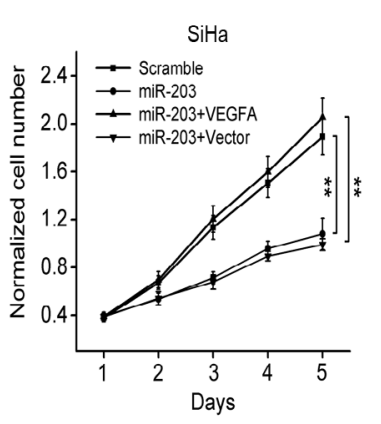



(C) 2013 S. Karger AG, Basel www.karger.com/cpb
C

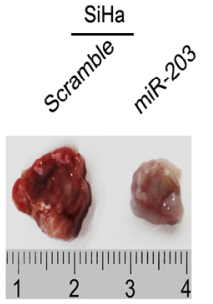

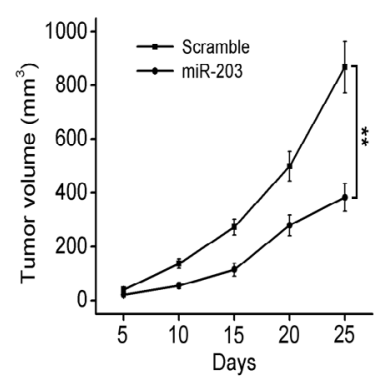
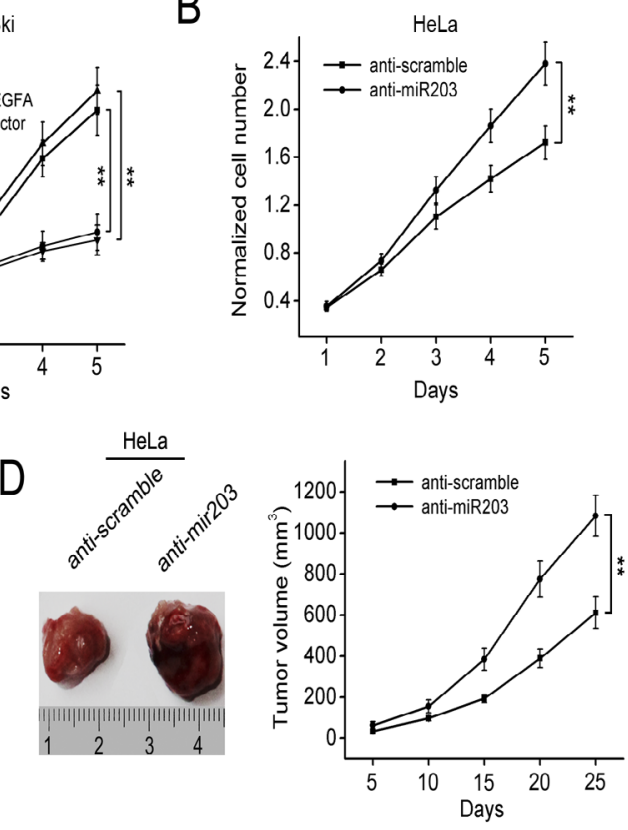

E
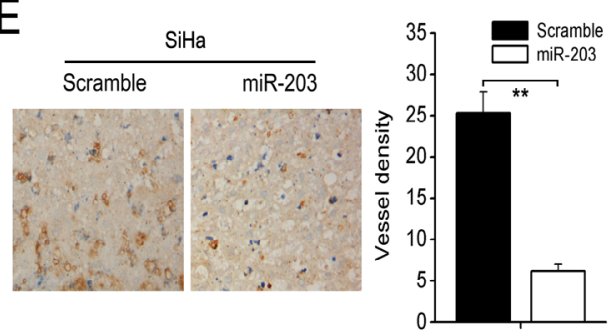

$\mathrm{F}$


Fig. 4. miR-203 suppressed cell proliferation, tumor growth, and angiogenesis. (A) miR-203 overexpression arrested cell proliferation, but this was rescued upon co-expression of VEGFA without 3'UTR in SiHa and CaSki cells. Data are presented as mean \pm standard error from 3 independent experiments. (B) Knockdown of miR-203 accelerated cell proliferation in HeLa cells. Data are presented as mean \pm standard error from 3 independent experiments. (C) miR-203 overexpression inhibited tumor growth of SiHa cells in mouse xenograft models. The left-hand panel contains a photograph of xenograft tumor masses from nude mice. The right-hand panel shows the volume of the xenograft tumors. Data represent mean \pm standard error from 8 SCID mice. (D) miR-203 knockdown promoted tumor growth of HeLa cells. Data represent mean \pm standard error from 8 SCID mice. (E, F) Representative tumor sections stained by antibody against human CD31. The results are presented as mean \pm standard error $(\mathrm{n}=8)$. Magnification, $\times 200{ }^{*} p<0.05$ and ${ }^{* *} p<0.01$.

normal cervical samples via qRT-PCR. We found that VEGFA expression in cervical cancer was significantly higher than in normal cervical tissue samples (Fig. 3F). We evaluated the correlation of VEGFA expression to that of miR-203 in the 34 cervical cancer tissue samples and identified a significant reverse correlation $\left(\mathrm{R}^{2}=0.708 ; p<0.001 ;\right.$ Fig. $\left.3 \mathrm{G}\right)$.

miR-203 suppressed cervical cancer cell proliferation and angiogenesis by targeting VEGFA

We explored the biological role of miR-203 in cervical cancer tumorigenesis and found that miR-203 overexpression markedly suppressed the growth of SiHa and CaSki cells and that re-expression of VEGFA (without an endogenous 3'-UTR) rescued this inhibition (Fig. 4A). Meanwhile, miR-203 depletion promoted HeLa cell growth (Fig. 4B). To confirm the antitumor effects of miR-203 in vivo, we implanted SiHa cells either overexpressing miR-203 or scrambled controls in the right flanks of nude mice. As shown in Fig. 4C, tumor growth was 
significantly reduced when miR-203 was stably expressed in SiHa cells. Accordingly, the miR203 knockdown tumors induced by HeLa cells were smaller in size compared to the scramble control tumors. miR-203 transfection-derived tumors were also found to have much smaller and fewer blood vessels compared to those of the control group (Fig. 4E), whereas miR-203 knockdown tumors had more blood vessels (Fig. 4F). These results indicate that miR-203 is able to suppress the growth of cervical cancer cells.

\section{Discussion}

miRNAs play key roles in cancer development and progression [23-25]. Recent studies found that miR-203 was significantly deregulated in cervical carcinoma [11-13]; however, the underlying mechanisms by which miR-203 affects tumor growth and angiogenesis are still not well elucidated. In the present study, we confirmed that miR-203 expression levels were significantly down-regulated in cervical cancer tissues and cell lines. Interestingly, miR-203 expression patterns differ among human cancers. miR-203 down-regulation was described in mucosa-associated lymphoid tissue lymphoma, central nervous system tumors, and hepatocellular carcinoma [26-28], whereas its up-regulation was shown in colon and ovarian cancers $[29,30]$. These controversial results suggest that the role of miR-203 is possibly tumor specific and highly dependent on its targets in different cancers.

Dysregulation of miRNAs expression occurs through multiple mechanisms including promoter methylation [31]. Recently, hypermethylation of miR-203 promoter has been found in HCC, cervical cancer and CML $[12,13,17,32]$. In this study, we confirmed that the mechanism of miR-203 downregulation was closely associated with the hypermethylation in its promoter region. Full or partial methylation was observed in cervical cancer cell lines and tissues by MSP analysis. Bisulfite genomic sequencing (BGS) of the miR-203 promoter region confirmed dense methylation in cervical cancer cell lines; however, very few methylation was detected in the normal cervical tissues. Furthermore, the reduced expression of miR203 could be restored by the treatment of demethylation agent 5-aza-2'-deoxycytidine (Aza), indicating that promoter methylation was the major mechanism of the downregulation of miR-203 in cervical cancer.

We further explored the possible targets of miR-203 in cervical cancer cells through different computational algorithms. Among the candidate target genes, we focused on VEGFA because of its role as a regulator of tumor growth and angiogenesis. Using a dual-luciferase reporter assay we showed that miR-203 directly bound to the 3'-UTR of VEGFA, which contains a miR-203-binding site. miR-203 overexpression significantly down-regulated VEGFA expression at the mRNA and protein levels. We observed substantial up-regulation of VEGFA mRNA in cervical cancer tissues and miR-203 levels were negatively correlated with VEGFA levels. Collectively, these results confirmed that miR-203 directly targeted VEGFA in cervical cancer.

To reveal the role of miR-203 in cervical cancer, we evaluated the effects of miR-203 on cell proliferation, tumor growth, and angiogenesis. We found that miR-203 overexpression markedly suppressed cell proliferation, whereas miR-203 deletion by anti-miR2 03 promoted cell growth. Re-expression of VEGFA in miR-203-overexpressed cells reversed the effects of miR-203, suggesting an anti-proliferative role of miR-203 attributable largely to VEGFA down-regulation. miR-203 overexpression suppressed cervical cancer cell tumorigenicity and angiogenesis in nude mouse xenograft models. To the best of our knowledge, several recent studies have reported the tumor-suppressor role of miR-203 in cancers [14-17]. Liu et al. [14] showed that miR-203 suppressed bladder cancer development by repressing B-cell leukemia/lymphoma-w expression. Melino et al. [16] found that miR-203 controlled proliferation, migration, and invasive potential of prostate cancer cell lines. Notably, miR203 has been reported to suppress epithelial to mesenchymal transition (EMT) in several tumor cell lines, such as prostate and breast cancer [16, 33]. Given that colon cancer cells undergoing EMT displayed increased VEGF secretion [34], we speculate that increased VEGF 
expression via downregulation of miR-203 in cervical cancer cell lines may be part of an EMT program. However, this issue awaits further investigation.

In summary, our data suggest that miR-203 suppressed tumor growth and angiogenesis by directly targeting VEGFA expression in cervical cancer. We identified a link between miR203 and the VEGFA axis, which apparently plays a vital role in cervical cancer angiogenesis. Thus, the miR-203/VEGFA pathway presents a viable candidate target for cervical cancer treatment.

\section{References}

1 Hu X, Schwarz JK, Lewis JS Jr, Huettner PC, Rader JS, Deasy JO, Grigsby PW, Wang X: A microRNA expression signature for cervical cancer prognosis. Cancer Res 2010;70:1441-1448.

$>2$ Ambros V: The functions of animal microRNAs. Nature 2004;431:350-355.

-3 Miska EA: How microRNAs control cell division, differentiation and death. Curr Opin Genet Dev 2005; 15:563-568.

4 Calin GA, Croce CM: MicroRNA signatures in human cancers. Nat Rev Cancer 2006;6:857-866.

5 Johnson CD, Esquela-Kerscher A, Stefani G, Byrom M, Kelnar K, Ovcharenko D, Wilson M, Wang X, Shelton J, Shingara J, Chin L, Brown D, Slack FJ: The let-7 microRNA represses cell proliferation pathways in human cells. Cancer Res 2007;67:7713-7722.

6 Kent OA, Mendell JT: A small piece in the cancer puzzle: microRNAs as tumor suppressors and oncogenes. Oncogene 2006;25:6188-6196.

7 Qiang R, Wang F, Shi LY, Liu M, Chen S, Wan HY, Li YX, Li X, Gao SY, Sun BC, Tang H: Plexin-B1 is a target of miR-214 in cervical cancer and promotes the growth and invasion of HeLa cells. Int J Biochem Cell Biol 2011;43:632-641.

8 Liu L, Yu X, Guo X, Tian Z, Su M, Long Y, Huang C, Zhou F, Liu M, Wu X, Wang X: MiR-143 is downregulated in cervical cancer and promotes apoptosis apoptosis and inhibits tumor formation by targeting Bcl-2. Mol Med Report 2011;5:753-760.

-9 Wang F, Li Y, Zhou J, Xu J, Peng C, Ye F, Shen Y, Lu W, Wan X, Xie X: MiR-375 is down-regulated in squamous cervical cancer and inhibits cell migration and invasion via targeting transcription factor SP1. Am J Pathol 2011;179:2580-2588.

10 Au Yeung CL, Tsang TY, Yau PL, Kwok TT: Human papillomavirus type 16 E6 induces cervical cancer cell migration through the p53/microRNA-23b/urokinase-type plasminogen activator pathway. Oncogene 2011;30:2401-2410.

11 Lee JW, Choi CH, Choi JJ, Park YA, Kim SJ, Hwang SY, Kim WY, Kim TJ, Lee JH, Kim BG, Bae DS: Altered MicroRNA expression in cervical carcinomas. Clin Cancer Res 2008;14:2535-2542.

$>12$ Botezatu A, Goia-Rusanu CD, Iancu IV, Huica I, Plesa A, Socolov D, Ungureanu C, Anton G: Quantitative analysis of the relationship between microRNA-124a, -34b and -203 gene methylation and cervical oncogenesis. Mol Med Rep 2011;4:121-128.

$>13$ Wilting SM, Verlaat W, Jaspers A, Makazaji NA, Agami R, Meijer CJ, Snijders PJ, Steenbergen RD: Methylation-mediated transcriptional repression of microRNAs during cervical carcinogenesis. Epigenetics 2013;8:220-228.

>14 Bo J, Yang G, Huo K, Jiang H, Zhang L, Liu D, Huang Y: microRNA-203 suppresses bladder cancer development by repressing bcl-w expression. FEBS J 2011;278:786-792.

15 Wang C, Zheng X, Shen C, Shi Y: MicroRNA-203 suppresses cell proliferation and migration by targeting BIRC5 and LASP1 in human triple-negative breast cancer cells. J Exp Clin Cancer Res 2012;31:58.

-16 Viticchiè G, Lena AM, Latina A, Formosa A, Gregersen LH, Lund AH, Bernardini S, Mauriello A, Miano R, Spagnoli LG, Knight RA, Candi E, Melino G: MiR-203 controls proliferation, migration and invasive potential of prostate cancer cell lines. Cell Cycle 2012;10:1121-1131.

17 Furuta M, Kozaki KI, Tanaka S, Arii S, Imoto I, Inazawa J: miR-124 and miR-203 are epigenetically silenced tumor-suppressive microRNAs in hepatocellular carcinoma. Carcinogenesis 2010;31:766-776. 


\begin{tabular}{|c|c|c|}
\hline Cellular P & Cell Physiol Biochem 2013;32:64-73 & \\
\hline and Biochemistry & $\begin{array}{l}\text { DOI: 10.1159/000350125 } \\
\text { Publisned online: July 04, } 2013\end{array}$ & $\begin{array}{l}\text { O } 2013 \text { S. Karger AG, Basel } \\
\text { www.karger.com/cpb }\end{array}$ \\
\hline
\end{tabular}

18 Frommer M, McDonald LE, Millar DS, Collis CM, Watt F, Grigg GW, Molloy PL, Paul CL: A genomic sequencing protocol that yields a positive display of 5-methylcytosine residues in individual DNA strands. Proc Natl Acad Sci USA 1992;89:1827-1831.

19 Saito Y, Liang G, Egger G, Friedman JM, Chuang JC, Coetzee GA, Jones PA: Specific activation of microRNA-127 with downregula-tion of the proto-oncogene BCL6 by chromatin-modifying drugs in human cancer cells. Cancer Cell 2006;9:435-443.

20 Xu J, Li Y, Wang F, Wang X, Cheng B, Ye F, Xie X, Zhou C, Lu W: Suppressed miR-424 expression via upregulation of target gene Chk1 contributes to the progression of cervical cancer. Oncogene 2013;32:976987.

21 Ferrara N, Gerber HP, LeCouter J: The biology of VEGF and its receptors. Nat Med 2003;9:669-676.

-22 Roskoski R Jr: Vascular endothelial growth factor (VEGF) signaling in tumor progression. Crit Rev Oncol Hematol 2007;62:179-213.

23 Wang X, Tang S, Le SY, Lu R, Rader JS, Meyers C, Zheng ZM: Aberrant expression of oncogenic and tumorsuppressive microRNAs in cervical cancer is required for cancer cell growth. PLoS One 2008;3:e2557.

24 Kang HW, Wang F, Wei Q Zhao YF, Liu M, Li X, Tang H: miR-20a promotes migration and invasion by regulating TNKS2 in human cervical cancer cells. FEBS Lett 2012;586:897-904.

25 Pang RT, Leung CO, Ye TM, Liu W, Chiu PC, Lam KK, Lee KF, Yeung WS: MicroRNA-34a suppresses invasion through downregulation of Notch1 and Jagged1 in cervical carcinoma and choriocarcinoma cells. Carcinogenesis 2010;31:1037-1044.

-26 Craig VJ, Cogliatti SB, Rehrauer H, Wündisch T, Müller A: Epigenetic silencing of microRNA-203 dysregulates ABL1 expression and drives Helicobacter-associated gastric lymphomagenesis. Cancer Res 2011;71:3616-3624.

-27 Gaur A, Jewell DA, Liang Y, Ridzon D, Moore JH, Chen C, Ambros VR, Israel MA: Characterization of microRNA expression levels and their biological correlates in human cancer cell lines. Cancer Res 2007;67:2456-2468.

28 [28] Ladeiro Y, Couchy G, Balabaud C, Bioulac-Sage P, Pelletier L, Rebouissou S, Zucman-Rossi J: MicroRNA profiling in hepatocellular tumors is associated with clinical features and oncogene/tumor suppressor gene mutations. Hepatology 2008;47:1955-1963.

-29 Schetter AJ, Leung SY, Sohn JJ, Zanetti KA, Bowman ED, Yanaihara N, Yuen ST, Chan TL, Kwong DL, Au GK, Liu CG, Calin GA, Croce CM, Harris CC: MicroRNA expression profiles associated with prognosis and therapeutic outcome in colon adenocarcinoma. JAMA 2008;299:425-436.

-30 Iorio MV, Visone R, Di Leva G, Donati V, Petrocca F, Casalini P, Taccioli C, Volinia S, Liu CG, Alder H, Calin GA, Ménard S, Croce CM: MicroRNA signatures in human ovarian cancer. Cancer Res 2007;67:8699-8707.

-31 Hiroki E, Suzuki F, Akahira J, Nagase S, Ito K, Sugawara J, Miki Y, Suzuki T, Sasano H, Yaegashi N: MicroRNA$34 \mathrm{~b}$ functions as a potential tumor suppressor in endometrial serous adenocarcinoma. Int J Cancer 2012;131:E395-404.

-32 Bueno MJ, Pérez de Castro I, Gómez de Cedrón M, Santos J, Calin GA, Cigudosa JC, Croce CM, FernándezPiqueras J, Malumbres M: Genetic and epigenetic silencing of microRNA-203 enhances ABL1 and BCRABL1 oncogene expression. Cancer Cell 2008;13:496-506.

-33 Ding X, Park SI, McCauley LK, Wang CY: Signaling between transforming growth factor $\beta$ (TGF- $\beta$ ) and transcription factor SNAI2 represses expression of microRNA miR-203 to promote epithelial-mesenchymal transition and tumor metastasis. J Biol Chem 2013;288:10241-10253.

-34 Sakuma K, Aoki M, Kannagi R: Transcription factors c-Myc and CDX2 mediate E-selectin ligand expression in colon cancer cells undergoing EGF/bFGF-induced epithelial-mesenchymal transition. Proc Natl Acad Sci USA 2012;109:7776-7781. 